\title{
Adenovirus-mediated cancer gene therapy and virotherapy (Review)
}

\author{
TAKUYA FUKAZAWA ${ }^{1}$, JUNJI MATSUOKA $^{1,2}$, TOMOKI YAMATSUJI $^{1}$, \\ YUTAKA MAEDA $^{3}$, MARY L. DURBIN ${ }^{4}$ and YOSHIO NAOMOTO ${ }^{1}$ \\ Departments of ${ }^{1}$ Gastroenterological Surgery, ${ }^{2}$ Palliative Medicine, Okayama University \\ Graduate School of Medicine, Dentistry and Pharmaceutical Sciences, Okayama, Japan 700-8558; \\ ${ }^{3}$ Division of Pulmonary Biology, Cincinnati Children's Hospital Medical Center, \\ Cincinnati, OH 45229-3039; ${ }^{4}$ Department of Ecology and Evolutionary Biology, \\ University of California, Irvine, CA 92697-2525, USA
}

Received September 9, 2009; Accepted October 21, 2009

DOI: 10.3892/ijmm_00000306

\begin{abstract}
Gene therapy and virotherapy are among the approaches currently used to treat malignant tumors. Gene therapy and virotherapy use a specific therapeutic gene that causes death in cancer cells. In early attempts at gene therapy, therapeutic genes were driven by ubiquitous promoters such as the CMV promoter, which induce non-specific toxicity to normal cells and tissues in addition to the cancer cells. Recently, novel cancer- and/or tissue-specific promoter systems have been developed to target cancer cells but not normal cells including stem cells. In this review, we describe cancer and/or tissue-specific gene therapy systems for the treatment of cancer. In particular, we will discuss three systems for gene therapy and virotherapy: i) tissue-specific promoter systems, ii) cancer-specific promoter systems, and iii) oncolytic virotherapy. We will also discuss the major challenges of cancer-targeting vector systems and future directions in this area.
\end{abstract}

\section{Contents}

1. Introduction

2. Tissue-specific promoter systems

3. Cancer-specific promoter systems

4. Oncolytic virotherapy

5. Major challenges of cancer-targeting vector systems

6. Conclusions

Correspondence to: Dr Takuya Fukazawa, Department of Gastroenterological Surgery, Okayama University Graduate School of Medicine, Dentistry and Pharmaceutical Sciences, 2-5-1 Shikata-cho, Okayama 700-8558, Japan

E-mail: fukazawat@aol.com

Key words: adenovirus, specific promoter, gene therapy, virotherapy

\section{Introduction}

Gene therapy and virotherapy are methods to introduce therapeutic genes into cancer cells for the treatment of cancer. The adenoviral vector has been used as a transfer vehicle to introduce genes into cancer cells since it is more efficient than non-viral gene transfer methods (e.g. cationic polymerDNA complexes) $(1,2)$. The adenoviral vector is stable in vivo and efficient in gene delivery to both dividing and nondividing cells and rarely causes any significant disease itself $(3,4)$. However, one of the limitations of gene therapy using the adenoviral vector is the non-specific expression of therapeutic genes in normal cells causing toxicity to non-cancerous tissues. Targeted expression of therapeutic genes is essential to prevent this toxicity. Recent understanding of tissue- and cancer-specific gene regulation in organogenesis and cancer fields has enabled the development of new promoter/enhancer systems to express therapeutic genes only in targeted cells and tissues. Cancer-specific therapeutic gene expression reduces undesirable toxicity. Thus, adenoviral vectors carrying a cancer-specific promoter system driving a therapeutic gene that is expressed only in cancer cells could be a means to safeguard against unwanted transduction to normal cells and tissues $(5,6)$.

\section{Tissue-specific promoter systems}

The early adenoviral vectors targeted, not only cancer cells, but also normal cells and tissues since constitutive promoters such as cytomegalovirus (CMV) and Rous sarcoma virus (RSV) were used to drive therapeutic genes (7). The expression of therapeutic genes in unintended, non-targeted normal tissues potentially causes undesirable toxic side effects. To reduce this toxicity, cancer or tissue-specific promoter systems have been developed by replacing constitutive promoters with promoters/enhancers of cancer/tissuespecific gene markers in the adenovirus vector. In this section, we describe tissue-specific promoter systems that target cancers originating from different tissues including prostate, lung, breast, myeloma, and pancreatic cancers. 
To target prostate cancer, several prostate tissue-specific promoters including prostate-specific antigen (PSA), probasin (PB) and prostate-specific membrane antigen (PSMA) have been tested for their specificity (8-10). Shi et al (11) reported that a helper-dependent adenoviral vector expressing the luciferase gene driven by the PSA promoter/enhancer effectively transduced and expressed luciferase in PSApositive prostate cancer LNCaP cells. Wu et al (12) generated an adenoviral vector expressing the luciferase gene driven by the chimeric PSA enhancer (Ad-PSE-BC-luc). Systemic administration of Ad-PSE-BC-luc into xenografted SCID mice with LAPC-9 human prostate cancer cells resulted in prostate cancer specific luciferase expression while generalized expression occurred using the cytomegalovirus (CMV) promoter driven vector (Ad-CMV-luc). Probasin is also a prostate-specific antigen. Andriani and colleagues (13) generated an adenoviral vector expressing an apoptotic Bax gene driven by the probasin promoter modified to contain two androgen response elements (Av-ARR2PB-Bax). The androgen dihydrotestosterone induced Bax-mediated apoptosis in $\mathrm{LNCaP}$ prostate cancer cells after Av-ARR2PB-Bax infection. The vector also exerted an antitumor effect in LNCaP xenograft tumors. Furuhata et al (14) generated an adenoviral vector expressing HSV-tk driven by a modified rat probasin $(\mathrm{rPB})$ promoter and found significant growth suppression of the androgen-independent prostate cancer cells in the presence of the prodrug ganciclovir in vivo. The modified $\mathrm{rPB}$ promoter is able to drive therapeutic genes in response to retinoid in both androgen-dependent and androgen-independent prostate cancer cells and not in other cancer cells or in normal cells.

To target lung cancer cells, the promoters of several types of surfactant protein genes have been tested. Surfactant protein A (SP-A) is expressed in a high proportion of NSCLC tumors with expression limited to the respiratory epithelium (15-18). SP-A can be used as one of the tissue-specific promoters to target lung cancer. Smith et al (19) reported that, in plasmid transduction experiments, a luciferase plasmid driven by the 2.75-kb upstream region of the SP-A gene produced significant luciferase activity in SP-A-producing pulmonary H441 adenocarcinoma cells, but only background levels in non-SPA-producing A549 adenocarcinoma cells. Moreover, the plasmid vector containing HSV-tk driven by the SP-A promoter showed cytotoxic effects in $\mathrm{H} 441$ cells but much less in A549 cells. Thus, SP-A promoter elements can be useful for directing the specific expression of a therapeutic gene for the treatment of lung cancer. Surfactant protein B (SP-B) expression is also limited to lung cells (20-23). Strayer et al (24) showed that an adenoviral vector that expresses the lacZ gene driven by the 5' flanking region of the SP-B promoter (rAd.SPB.lacZ) targeted H441 and A549 adenocarcinoma cells but not HeLa cervical cancer cells and primary human fetal lung fibroblasts. In contrast, an adenoviral vector that expresses the lacZ gene driven by the constitutive RSV promoter (rAd.RSV.lacZ) targeted all the cells tested.

The MUC1/DF3 promoter has been used to target multiple cancers, including breast cancer, myeloma and pancreatic cancer. MUC1/DF3 is a transmembrane mucin gene normally expressed on the apical borders of secretory epithelial cells.
Moehle et al (25) found that MUC1 was highly expressed in adult prostate, mammary glands, trachea, lung, small intestine, colon, and in fetal lung. Chen and colleagues (26) generated recombinant adenoviral vectors containing the 5' flanking region of the MUC1/DF3 promoter $(-725$ to +31$)$, expressing the LacZ gene (Ad.DF3ß-gal) or the herpes simplex virus thymidine kinase (HSV-tk) gene (Ad.Df3-tk). The authors showed that these vectors induced the target gene in MUC1/ DF3-positive breast cancer cells but not in MUC1/DF3negative breast carcinoma cells. Moreover, intraperitoneal injection of Ad.DF3-tk followed by ganciclovir (GCV) treatment resulted in inhibition of tumor growth in an intraperitoneal breast cancer metastases model. Teoh and colleagues (27) analyzed the expression of DF3/MUC1 and the adenovirus receptor CAR in multiple myeloma cells by flow cytometry. They confirmed that the majority of myeloma cells expressed DF3/MUC1 and CAR while normal bone marrow did not express CAR, indicating that an adenovirus vector using the promoter of the DF3/MUC1 gene targets only myeloma and not normal bone marrow. Transduction with the tk gene driven by Ad.DF3-tk followed by treatment with GCV purged multiple myeloma OCI-My5 and RPMI 8226 cells within bone marrow mononuclear cells. Chen et al (28) also generated an adenoviral vector expressing the human somatostatin receptor subtype 2 (hSSTR2) gene driven by the 786-bp upstream region of the DF3/MUC1 gene (AdMUC1-hSSTR2). They demonstrated that there was significant inhibition of cell proliferation in DF3/MUC1positive pancreatic cancer Panc-1 cells. These results demonstrate that utilization of the MUC1/DF3 promoter in an adenoviral vector confers selective expression of target genes in breast cancer, multiple myeloma and pancreatic cancer.

Tissue-specific promoter systems are useful for targeting differentiated cancer. Such systems are not supposed to target normal proliferating tissue, including stem cells. However, there is always a risk of targeting normal differentiated tissue. In the next section, we will discuss another system that targets proliferating cells but not normal differentiated cells.

\section{Cancer-specific promoter systems}

Five cancer-specific promoter systems utilize genes that are highly expressed in cancer: carcinoembryonic antigen (CEA), $\alpha$-fetoprotein (AFP), midkine (MK), survivin, and telomerase.

Carcinoembryonic antigen (CEA) is known as an oncofetal protein and adhesion molecule in tumorigenesis. CEA is not usually expressed in normal tissue but it is present in various tumors including gastrointestinal, lung or breast cancer $(29,30)$. The CEA promoter is considered a candidate for gene therapy targeting CEA-positive cancer cells. The cisacting elements of the CEA promoter for cell-type specific expression were characterized by Schrewe and colleagues (31). Tanaka et al (32) constructed a recombinant adenoviral vector expressing HSV-tk driven by the CEA promoter (AdCEA/tk). The cell growth of CEA-producing MKN28 and MKN45 gastric cancer cells infected with AdCEA/tk in the presence of GCV were significantly suppressed while 
non-CEA-producing MKN1 gastric cancer cells and HeLa cervical cancer cells infected with AdCEA/tk remained resistant to GCV. Goto et al (33) also developed two recombinant adenoviral vectors: one containing the Cre gene under the control of the CEA promoter (AdCEA-Cre) and the other containing the HSV-tk gene (Ad.lox-TK). Coinfection of AdCEA-Cre and Ad.lox-TK followed by GCV administration significantly suppressed the CEA-producing tumor cell-derived peritonitis carcinomatosa in a mouse model. Adenoviral vectors driven by the CEA promoter have been used to target other CEA producing cancers including lung cancer and breast cancer $(34,35)$.

Hepatocellular carcinoma (HCC) is one of the most common cancers in the world. For the majority of patients, the current treatments remain unsatisfactory and the prognosis is poor. $\alpha$-fetoprotein (AFP) is another oncofetal protein. An elevated level of serum AFP is observed in patients with $\mathrm{HCC}$ at an advanced stage. Thus, it is one of the diagnostic and prognostic markers of HCC $(36,37)$. Several cis- and trans-acting elements regulating the human AFP gene have been characterized. Hepatocyte-specific enhancers exist in a distant upstream region $(-4.0 \mathrm{~kb}$ and $-3.3 \mathrm{~kb})$ of the AFP gene $(38,39)$. A hepatocyte-specific silencer was located between the enhancer region and the hepatocyte-specific promoter region (40). Kanai and colleagues (41) developed a recombinant adenovirus expressing the HSV-tk gene containing the human AFP enhancer domain (-4.0 kb and -3.3 kb) and $170 \mathrm{bp}$ of the AFP promoter (Ad.AFPtk) to target AFP-producing HCC cells. The Ad.AFPtk construct along with GCV reduced the cell growth of AFP-producing HuH7 and HepG2 HCC cells but did not affect non-AFP producing HLF HCC cells. Treatment with a recombinant adenovirus expressing the Escherichia coli cytosine deaminase (CD) gene driven by the human AFP promoter/enhancer (AdAFPCD) with 5FC also regressed AFP-producing $\mathrm{HuH} 7$ and HepG2 HCC cell xenografts (42). Miao et al (43) constructed a recombinant adenovirus-Ad/AFPtBid that contained a tBid gene (truncated BH3-interacting domain death agonist) driven by an AFP promoter. Ad/AFPtBid induced apoptosis both in p53sensitive $\mathrm{HCC}$ PLC/PRF/5 cells and p53-resistant HCC Hep3B cells. In in vivo experiments, intratumor injection of Ad/AFPtBid significantly inhibited tumor growth of Hep3B cell xenograft tumors in nude mice. These results show the efficacy of the AFP promoter in cancer gene therapy against AFP-producing hepatocellar carcinoma.

Midkine (MK) is a heparin-binding growth factor identified as a product of a retinoic acid-responsive gene. MK has mitogenic activity, anti-apoptotic activity, and angiogenic activity. MK is also involved in oncogenic transformation (44-49). Many human malignant tumors express high levels of MK protein while normal human tissues including liver do not $(50)$. Adachi et al $(51,52)$ developed a recombinant adenoviral vector containing the herpes simplex thymidine kinase gene under the control of the 2.3-kb upstream promoter of the human MK gene (AdMKTK). AdMKTK induced marked cell death in response to GCV in G-401 Wilms' tumor cells and SK-N-SH neuroblastoma cells, but did not induce liver toxicity. These findings suggest that the MK promoter may be an important tumor-specific promoter by virtue of its very low hepatic toxicity and high tumor activity.
Survivin is a member of the IAP gene family containing a single baculovirus IAP repeat and no RING finger (53). The expression of survivin is detected in various types of tumors, including non-Hodgkin's lymphoma, breast cancer, lung cancer, ovarian cancer, hepatocellular carcinoma, esophageal cancer and colorectal cancer (53-59) but not in normal tissues including the liver $(53,60,61)$. The survivin promoter is highly active in breast cancer, lung cancer, ovarian cancer and colorectal cancer but inactive in human liver tissue (62-64). These findings suggest that the survivin promoter may be a good candidate to drive therapeutic gene expression for cancer gene therapy. Lu and colleagues (65) constructed recombinant adenoviral vectors expressing the luciferase gene under the control of four different promoters including that of survivin, Cox-2, CXCR4 or EGP-2. Luciferase activity was analyzed in multiple tumor cells including primary melanoma cells and human epithelial melanocytes (HEMs) after infection with these vectors. Among the four promoters, the survivin promoter generated the highest activities in melanoma cells and primary melanoma cells but not in HEMs. Moreover, the survivin promoter showed less activity in vivo in major mouse organs including the liver. These data suggest that the survivin promoter may be useful as a tumor-specific promoter with applications for transcriptional targeting of adenovirus vector-based cancer gene therapy.

Telomerase is an RNA-dependent DNA polymerase that synthesizes telomeric DNA. It consists of an RNA component (hTR), a catalytic protein subunit (hTERT), and a telomeraseassociated protein TEP1 $(66,67)$. Expression of hTERT is observed at high levels in malignant tumors. There is a strong correlation between hTERT expression and telomerase activity in various types of tumors (68). Takakura et al (69) identified an E-box (CACGTG) binding site on the hTERT promoter that is required for c-Myc-mediated hTERT promoter activation. Majumdar et al (70) constructed a recombinant adenoviral vector carrying the HSV-tk gene under the control of a 208-bp region upstream of the transcription initiation site of the hTERT promoter (AdhTERT/tk). They also constructed an adenovirus carrying the HSV-tk gene under the control of the constitutive CMV promoter (AdhTERT/tk) to compare with the efficacy of AdhTERT/tk. In a xenograft model using the human 143B osteosarcoma cells, a single injection of both AdhTERT/tk and $\mathrm{AdCMV} / \mathrm{tk}$ viruses resulted in equivalent tumor regression upon GCV treatment. However, expression of the HSV-tk gene was detected in the liver as well as in tumors in mice injected intratumorally with the AdCMV/tk adenovirus. Expression of the HSV-tk gene by AdCMV/tk in combination with GCV resulted in severe liver histopathology. In contrast, expression of the HSV-tk gene was detected only in tumors and not in the liver in mice with the AdhTERT/tk. These results indicate that the adenovirus system using the hTERT promoter targets only tumors and not normal tissues including liver. Bilsland et al (71) constructed adenoviral vectors harboring the suicide gene bacterial nitroreductase (NTR), which bioactivates the prodrug CB1954 into an active cytotoxic alkylating agent under the control of an 876-bp fragment of the hTR proximal promoter (Ad-hTR/NTR) or a 541-bp fragment of the hTERT promoter (Ad-hTERT/NTR). Western blot analysis indicated that NTR expression was 
detectable only in cancer cells but not in normal cells after Ad-hTR/NTR or Ad-hTERT/NTR infection. Seven of nine types of cancer cells resulted in up to 18 -fold sensitization to the prodrug CB1954 after Ad-hTR/NTR or Ad-hTERT/NTR infection while no sensitization was observed in four types of normal cells. Moreover, an antitumor effect was observed in cervical and ovarian xenograft models following a single intratumoral injection of these vectors, followed by injection with CB1954. These results indicate that the hTERT promoter is a tumor-specific promoter that may be useful for transcriptional targeting for cancer gene therapy.

It has been reported that stem cells, the lymphohematopoietic system, germ cells, and cirrhotic liver have significantly high telomerase activity (72-74). Therefore, the hTERT promoter-mediated gene expression system may cause unknown side effects in these cells and tissues. To develop a promoter system that targets only cancer cells, but not normal cells including stems cells, we recently designed a novel dual tissue- and cancer-specific promoter system by combining the hTERT promoter-mediated cancer-targeted system with a lung-specific tissue-targeted system [TTS system: thyroid transcription factor 1 (TTF1) gene under the control of the hTERT promoter and human surfactant protein A1 (hSPA1) promoter system] (75). We showed that the TTS construct is a lung cancer-specific adenoviral vector system expressing Bax that specifically induces cell death in lung cancer cells including gefitinib (epidermal growth factor receptor tyrosine kinase inhibitor)-resistant cells (6). Because normal cells also proliferate, it is not appropriate for cancer gene therapy to use a promoter such as hTERT that solely targets proliferating cells. Ideally, a promoter system should target cancer cells but not any type of normal cells. Identification and use of appropriate promoter systems that target specific cancer cell types and not normal cells is required for safe cancer gene therapy and virotherapy.

\section{Transcriptionally regulated oncolytic adenoviruses}

In early gene therapy attempts for the treatment of cancer, viral vectors were used that were modified to delete the function of self-replication in order to achieve safe gene transfer without inducing viral lysis to normal cells and tissues. However, the clinical trials of some suicide gene or corrective gene therapies revealed that the therapeutic effect of non-replicative viruses was limited $(76,77)$. In order to resolve this limitation, conditional replicative adenoviruses (CRAds) were developed for cancer gene therapy, which is also called virotherapy. The conditional replicative viruses are able to replicate and cause cell lysis only in the targeted tumor cells. In addition, the replicated viruses are able to infect neighboring cancer cells and continue this infection cycle until all of the tumor cells are eradicated (78). Virotherapy is the strategy of using a replication-competent virus for cancer therapy. For the safe use of virotherapy, the viruses must replicate only in targeted tumor cells but not in normal cells and tissues.

The ability of adenovirus to transform cells is dependent on the virus-encoded proteins, E1A and E1B, which induce cell cycle progression through the $\mathrm{S}$-phase and provide protection from apoptosis so that efficient virus replication can occur. The deletion of E1A causes the adenovirus to become susceptible to the antiviral mechanisms of the retinoblastoma $(\mathrm{Rb})$ protein by blocking the $\mathrm{G} 1$ to $\mathrm{S}$ transition. On the other hand, deletion of E1B allows p53 to induce apoptosis in infected cells, aborting replication and spread of the virus because the E1B $55 \mathrm{~K}$ protein binds $\mathrm{p} 53$, targeting it for degradation (79). ONYX-015 is an E1B 55K-deficient adenovirus that contains another viral E1A protein. The ONYX-015 virus is the first conditionally replicating adenovirus that has been tested in a variety of tumor types. Initial preclinical studies demonstrated that ONYX-015 effectively decreased tumor size $(80,81)$. Phase II clinical trials in head and neck cancer patients where intratumoral injection of ONYX-015 in combination with cisplatin and 5-fluorouracil showed a $63 \%$ overall response rate and with $27 \%$ of patients demonstrating a full clinical response (82).

Another approach for tumor selective virotherapy is the development of an adenoviral vector expressing E1A or/and E1B genes driven by tumor- or tissue-specific promoters and enhancers. This has been achieved in prostate cancer therapies by using the prostate-specific antigen (PSA) promoter. Rodriguez et al (83) generated an adenovirus expressing E1A under the control of the 5' flanking region of the human PSA gene (consisting of the enhancer at base pairs -5322 to -3738 fused to the PSA promoter at base pairs -541 to +12 ) and termed CN706. Immunoblot analysis showed that E1A expression was high in human PSA-producing LNCaP cells but not in PSA non-producing DU145 cells after CN706 infection. The titer of CN706 was significantly higher in LNCaP cells compared to other human PSA non-producing cells (HBL100, PANC1, MCF7, DU145, and OVCAR3). CN706 destroyed LNCaP cell-derived tumors and abolished PSA production in nu/nu mouse xenograft models after a single intratumoral injection. For the treatment of patients with locally recurrent prostate cancer after radiation therapy, a phase I dose-ranging study using an intra-prostatic injection of CV706 resulted in virus dose-dependent reductions in serum PSA levels in treated patients (84).

As we described above, the survivin promoter is a candidate for transcriptional targeting of cancer gene therapy. CRAds containing the survivin promoter have been used to treat glioma and mesothelioma. For the treatment of gliomas, Van Houdt et al (85) generated conditionally replicative adenoviruses expressing E1A under the control of the 5' flanking region of the human survivin promoter (a short segment -230 to -130 or a longer segment -1430 to 130 ) and termed them CRAd-S-S and CRAd-S-L, respectively. These adenoviruses efficiently replicated and killed a variety of established glioma tumor cells but were inactive in a normal human liver organ culture. Furthermore, these CRAds significantly inhibited the growth of glioma xenografts in vivo (85). Mesothelioma is a highly malignant neoplasm with no effective treatment. Zhu et al (60) constructed a conditionally replicative adenovirus regulated by the survivin promoter with a capsid modification (RGD or F5/3) in the adenovirus fiber region. These CRAd agents effectively targeted human mesothelioma cells and induced strong cytotoxicity in these cells in vitro and viral replication in a H226 murine xenograft model in vivo. These results suggest that the survivin-based CRAds are promising agents for targeting mesothelioma with 
low host toxicity. Together these data indicate that the survivin promoter is a promising tumor-specific promoter for transcriptional targeting of CRAds for cancer.

The cancer-specific midkine promoter has also been used to construct CRAds. Toyoda and colleagues (86) generated the CRAds driven by the $0.6-\mathrm{kb}$ midkine promoter (Ad5MK). Ad5MK showed more cytotoxicity for midkine-expressing Suit-2 and PANC-1 pancreatic cancer cells than for midkinenegative MIAPaCa-2 pancreatic cancer cells. In the Suit-2 cell-derived intraperitoneal xenograft mouse model, the Ad5MK-treated group survived significantly longer than control groups. These studies suggest that a midkine promoterbased conditionally replicative adenovirus might be a promising new virotherapy for pancreatic cancer.

In our own research we also used CRAd to target mesothelioma $(6,75,87)$. Gordon and colleagues $(88)$ profiled the gene expression pattern of malignant pleural mesothelioma, normal lung, and pleural tissues using cDNA microarrays. They reported that the CRI1 (CREBBP/EP300 inhibitory protein 1) gene was a specific marker for malignant pleural mesothelioma. We made a construct containing four tandem repeats of the CRI1 promoter-138/-1 (CRI1-138 4x) and observed that the use of the tandem repeats caused significantly higher promoter activity in malignant pleural mesothelioma cells but little promoter activity in normal mesothelial cells and normal fibroblasts. We developed a CRAdexpressing E1A driven by the CRI1-138 4x promoter system (Ad-CRI-138 4x/E1A). Ad-CRI-138 4x/E1A induced viral proliferation and cell death only in a mesothelioma specific manner and showed antitumor effects in a mesothelioma xenograft mouse model (87). These results suggest that CRI1-138 4x is a promising promoter system to use in CRAd to target mesothelioma.

In earlier cancer gene therapies using adenovirus, antiapoptotic genes, such as Bax, tBid and HSV-tk were used to cause cell death in cancer cells. However, cell death occurred only in virus-injected cancer cells but not in the non-injected neighboring cancer cells. In order to solve this limitation, CRAds were developed, which spread to all cancer cells even non-injected ones. The development of cancer type-specific promoters is still required to avoid targeting normal cells, but viral proteins including E1A causing cell lysis/death to all cancer cells will be employed for future cancer gene therapy.

\section{Major challenges of cancer-targeting vectors}

One of the major challenges in adenovirus-mediated cancer gene therapy and virotherapy is poor transduction in human tumors. The reason for this poor transduction is that tumor cells have limited surface expression of the Ad5 primary receptor, the coxsackievirus and adenovirus receptor (CAR) that are necessary for transduction. To resolve the problem, several modifications to the adenovirus fiber have been performed. Wickham et al (89) constructed adenoviral vectors, which contain modifications to the adenoviral fiber coat protein that redirect virus binding to either $\alpha(v)$ integrin [AdZ.F(RGD)] or heparan sulfate [AdZ.F(pK7)] cellular receptors. They reported that AdZ.F(RGD) increased gene delivery to endothelial and smooth muscle cells expressing $\alpha(\mathrm{v})$ integrins and that AdZ.F(pK7) increased transduction 5- to 500-fold in multiple cell types lacking high levels of the adenoviral fiber receptor. Another approach is to create chimeric viruses, usually based on adenovirus serotype 5 (Ad5). The fiber or knob domain is replaced by that of another serotype Ad5 with adenovirus serotype 3 (Ad3). This virus showed CAR-independent infectivity $(90,91)$. Ulasov and colleagues (92) have shown that a chimeric Ad5/3 vector which contains the shaft of adenovirus serotype 5 and the knob of adenovirus serotype 3 can target the CD46 cellular receptor and increase transduction of glioma cells. Kanerva et al (93) reported that the Ad5/3 vector increased transduction of ovarian cancer cells. Similarly, adenoviral vectors constructed with the serotype 17 fiber (Ad17) improve transduction of airway epithelial tissue. Adenoviral vectors with serotype 35 (Ad35) improve transduction of hematopoietic cells $(94,95)$. Krasnykh et al (96) replaced the fiber and knob domains with bacteriophage T4 fibritin proteins. This modified adenovirus lacks the ability to interact with CAR and demonstrates much higher reporter gene expression in HEK293 cells expressing a 6-His-binding receptor.

Immune responses against the adenovirus drastically limit the vector transduction efficiency and the duration of transgene expression. In order to overcome this issue several strategies have been examined. Immunosuppressive agents including cyclosporin, FK506 or anti-CD4 monoclonal antibody have been found to enhance transgene expression of adenoviral vectors (97-99). Chirmule and colleagues (100) treated rhesus monkeys with CD40 ligand antibody (hu5C8) after inoculating the lung with adenoviral vector. Immunological analyses demonstrated suppression of adenovirus-induced lymphoproliferation and cytokine responses (IL-2, INF- $\gamma$, IL-4, and IL-10) in the hu5C8-treated rhesus monkeys. The hu5C8 treatment resulted in significant and prolonged inhibition of the adenovirus-specific humoral response well beyond the time when hu5C8 effects were no longer significant.

Alteration of the immunodominant epitopes of the adenoviral capsid is also helpful in evading immune responses. Covalent attachment of polymers to adenoviral capsid protein has been shown to curtail antibody-mediated virus neutralization. O'Riordan et al (101) showed that PEG-modified adenovirus can be protected from antibody neutralization in the lungs of mice with high antibody titers to adenovirus, suggesting that PEGylation improves the ability to administer adenoviral vectors. Fisher et al $(102,103)$ reported that incorporation of targeting ligands such as basic fibroblast growth factor and vascular endothelial growth factor on $\mathrm{N}$ (2-hydroxypropyl) methacrylamide (HPMA)-coated virus produces ligand-mediated CAR-independent binding and uptake into cells which have appropriate receptors. Serotype switching in vector construction is another method to evade the vector immune response in human gene therapy since the neutralizing immune response to adenovirus is serotypespecific. The replication-defective Ad11 or Ad35 vectors have been reported to elude preexisting adenoviral immunity $(104,105)$. In addition, Parks et al (106) showed that helper dependent adenoviral vectors elicited a limited cell-mediated immune response without causing significant liver damage and toxicity. Roberts and colleagues (107) have reported the construction of a novel hexon-chimeric adenoviral vector that circumvents preexisting anti-vector immunity. These new 
vectors combined with CRAds whose expression is driven by cancer cell-type specific promoters will enable the targeting and destruction of cancer cells without affecting normal cells including stem cells.

\section{Conclusions}

Adenoviral vectors are the most promising and widely used platform for gene therapy and virotherapy. However, there have been problems associated with their use. Recently researchers have been working to improve the efficacy of these vectors by focusing on improving three parts of the vector systems. One goal is to obtain specificity to cancer cells in order to avoid damaging normal cells. The promoter design to acquire cancer cell specificity has evolved from the earlier constitutive promoter system to the cancer/tissue targeted promoter systems. A second goal is to improve the means to induce cancer cell death. The modification of viral proteins to cause cell death/lysis in cancer cells while also promoting viral replication in other cancer cells but not in normal cells has been realized. This breakthrough will enable the targeting not only of neighboring cancer cells, but also metastasized cancer cells. A third goal is to improve transduction. The surface structure of adenovirus vectors has been modified to deliver therapeutic genes into cancer cells more efficiently. Furthermore, some of the modifications enable the virus to avoid the immune response of the patient. These cancerspecific adenovirus vectors equipped with an efficient delivery system are ready to be used immediately in in vivo mouse models and tested for clinical trials.

\section{References}

1. Wolfert MA, Schacht EH, Toncheva V, Ulbrich K, Nazarova O and Seymour LW: Characterization of vectors for gene therapy formed by self-assembly of DNA with synthetic block copolymers. Hum Gene Ther 7: 2123-2133, 1996.

2. Zhang YP, Sekirov L, Saravolac EG, et al: Stabilized plasmidlipid particles for regional gene therapy: formulation and transfection properties. Gene Ther 6: 1438-1447, 1999.

3. Berkner KL: Development of adenovirus vectors for the expression of heterologous genes. Biotechniques 6: 616-629, 1988.

4. Berkner KL: Expression of heterologous sequences in adenoviral vectors. Curr Top Microbiol Immunol 158: 39-66, 1992.

5. Check E: A tragic setback. Nature 420: 116-118, 2002.

6. Fukazawa T, Maeda Y, Durbin ML, et al: Pulmonary adenocarcinoma-targeted gene therapy by a cancer- and tissuespecific promoter system. Mol Cancer Ther 6: 244-252, 2007.

7. Tong X, Engehausen DG, Freund CT, et al: The efficacy of adenovirus-mediated gene therapy of ovarian cancer is enhanced by using the cytomegalovirus promoter. Anticancer Res 18: 719-725, 1998

8. Luke MC and Coffey DS: Human androgen receptor binding to the androgen response element of prostate specific antigen. J Androl 15: 41-51, 1994.

9. Luchman HA, Friedman HC, Villemaire ML, Peterson AC and Jirik FR: Temporally controlled prostate epithelium-specific gene alterations. Genesis 46: 229-234, 2008

10. Lee SJ, Kim HS, Yu R, et al: Novel prostate-specific promoter derived from PSA and PSMA enhancers. Mol Ther 6: 415-421, 2002.

11. Shi CX, Hitt M, Ng P and Graham FL: Superior tissue-specific expression from tyrosinase and prostate-specific antigen promoters/enhancers in helper-dependent compared with firstgeneration adenoviral vectors. Hum Gene Ther 13: 211-224 2002.

12. Wu L, Matherly J, Smallwood A, et al: Chimeric PSA enhancers exhibit augmented activity in prostate cancer gene therapy vectors. Gene Ther 8: 1416-1426, 2001.
13. Andriani F, Nan B, Yu J, et al: Use of the probasin promoter ARR2PB to express $\mathrm{Bax}$ in androgen receptor-positive prostate cancer cells. J Natl Cancer Inst 93: 1314-1324, 2001

14. Furuhata S, Ide H, Miura Y, Yoshida T and Aoki K: Development of a prostate-specific promoter for gene therapy against androgen-independent prostate cancer. Mol Ther 7: 366-374, 2003.

15. Gazdar AF, Linnoila RI, Kurita Y, et al: Peripheral airway cell differentiation in human lung cancer cell lines. Cancer Res 50: 5481-5487, 1990 .

16. Bejarano PA, Baughman RP, Biddinger PW, et al: Surfactant proteins and thyroid transcription factor-1 in pulmonary and breast carcinomas. Mod Pathol 9: 445-452, 1996.

17. O'Reilly MA, Nogee L and Whitsett JA: Requirement of the collagenous domain for carbohydrate processing and secretion of a surfactant protein, SP-A. Biochim Biophys Acta 969: 176-184, 1988.

18. Khoor A, Gray ME, Hull WM, Whitsett JA and Stahlman MT: Developmental expression of SP-A and SP-A mRNA in the proximal and distal respiratory epithelium in the human fetus and newborn. J Histochem Cytochem 41: 1311-1319, 1993.

19. Smith MJ, Rousculp MD, Goldsmith KT, Curiel DT and Garver RI Jr: Surfactant protein A-directed toxin gene kills lung cancer cells in vitro. Hum Gene Ther 5: 29-35, 1994.

20. Maeda Y, Hunter TC, Loudy DE, Dave V, Schreiber V and Whitsett JA: PARP-2 interacts with TTF-1 and regulates expression of surfactant protein-B. J Biol Chem 281: 9600-9606, 2006.

21. Tokieda K, Iwamoto HS, Bachurski C, et al: Surfactant proteinB-deficient mice are susceptible to hyperoxic lung injury. Am J Respir Cell Mol Biol 21: 463-472, 1999.

22. Bruno MA, Bohinski RJ, Carter JE, Foss KA and Whitsett JA: Structure and function of the mouse surfactant protein B gene. Am J Physiol 268: L381-L389, 1995.

23. Bohinski RJ, Di Lauro R and Whitsett JA: The lung-specific surfactant protein $\mathrm{B}$ gene promoter is a target for thyroid transcription factor 1 and hepatocyte nuclear factor 3 , indicating common factors for organ-specific gene expression along the foregut axis. Mol Cell Biol 14: 5671-5681, 1994.

24. Strayer MS, Guttentag SH and Ballard PL: Targeting type II and Clara cells for adenovirus-mediated gene transfer using the surfactant protein B promoter. Am J Respir Cell Mol Biol 18: $1-11,1998$.

25. Moehle C, Ackermann N, Langmann T, et al: Aberrant intestinal expression and allelic variants of mucin genes associated with inflammatory bowel disease. J Mol Med 84: 1055-1066, 2006.

26. Chen L, Chen D, Manome Y, Dong Y, Fine HA and Kufe DW: Breast cancer selective gene expression and therapy mediated by recombinant adenoviruses containing the DF3/MUC1 promoter. J Clin Invest 96: 2775-2782, 1995.

27. Teoh G, Chen L, Urashima M, et al: Adenovirus vector-based purging of multiple myeloma cells. Blood 92: 4591-4601, 1998.

28. Chen L, Liu Q, Qin R, et al: Amplification and functional characterization of MUC1 promoter and gene-virotherapy via a targeting adenoviral vector expressing the hSSTR2 gene in MUC1-positive Panc-1 pancreatic cancer cells in vitro. Int J Mol Med 15: 617-626, 2005.

29. Wagener C, Petzold P, Kohler W and Totovic V: Binding of five monoclonal anti-CEA antibodies with different epitope specificities to various carcinoma tissues. Int J Cancer 33: 469-475, 1984

30. Goldenberg DM, Sharkey RM and Primus FJ: Carcinoembryonic antigen in histopathology: immunoperoxidase staining of conventional tissue sections. J Natl Cancer Inst 57: 11-22, 1976.

31. Schrewe H, Thompson J, Bona M, et al: Cloning of the complete gene for carcinoembryonic antigen: analysis of its promoter indicates a region conveying cell type-specific expression. Mol Cell Biol 10: 2738-2748, 1990.

32. Tanaka T, Kanai F, Okabe S, et al: Adenovirus-mediated prodrug gene therapy for carcinoembryonic antigen-producing human gastric carcinoma cells in vitro. Cancer Res 56: 1341-1345, 1996.

33. Goto H, Osaki T, Kijima T, et al: Gene therapy utilizing the Cre/loxP system selectively suppresses tumor growth of disseminated carcinoembryonic antigen-producing cancer cells. Int J Cancer 94: 414-419, 2001.

34. Qiao J, Doubrovin M, Sauter BV, et al: Tumor-specific transcriptional targeting of suicide gene therapy. Gene Ther 9: $168-175,2002$. 
35. Scholz IV, Cengic N, Baker CH, et al: Radioiodine therapy of colon cancer following tissue-specific sodium iodide symporter gene transfer. Gene Ther 12: 272-280, 2005

36. Bellet DH, Wands JR, Isselbacher KJ and Bohuon C: Serum alpha-fetoprotein levels in human disease: perspective from a highly specific monoclonal radioimmunoassay. Proc Natl Acad Sci USA 81: 3869-3873, 1984

37. Shimada M, Takenaka K, Fujiwara Y, et al: Des-gammacarboxy prothrombin and alpha-fetoprotein positive status as a new prognostic indicator after hepatic resection for hepatocellular carcinoma. Cancer 78: 2094-2100, 1996.

38. Watanabe K, Saito A and Tamaoki T: Cell-specific enhancer activity in a far upstream region of the human alpha-fetoprotein gene. J Biol Chem 262: 4812-4818, 1987.

39. Nakabayashi H, Watanabe K, Saito A, Otsuru A, Sawadaishi K and Tamaoki T: Transcriptional regulation of alpha-fetoprotein expression by dexamethasone in human hepatoma cells. J Biol Chem 264: 266-271, 1989.

40. Nakabayashi H, Hashimoto T, Miyao Y, Tjong KK, Chan J and Tamaoki T: A position-dependent silencer plays a major role in repressing alpha-fetoprotein expression in human hepatoma. Mol Cell Biol 11: 5885-5893, 1991.

41. Kanai F, Shiratori Y, Yoshida Y, et al: Gene therapy for alphafetoprotein-producing human hepatoma cells by adenovirusmediated transfer of the herpes simplex virus thymidine kinase gene. Hepatology 23: 1359-1368, 1996.

42. Kanai F, Lan KH, Shiratori Y, et al: In vivo gene therapy for alpha-fetoprotein-producing hepatocellular carcinoma by adenovirus-mediated transfer of cytosine deaminase gene. Cancer Res 57: 461-465, 1997

43. Miao J, Chen GG, Chun SY, et al: Adenovirus-mediated tBid overexpression results in therapeutic effects on p53-resistant hepatocellular carcinoma. Int J Cancer 119: 1985-1993, 2006.

44. Dai LC: Midkine translocated to nucleoli and involved in carcinogenesis. World J Gastroenterol 15: 412-416, 2009.

45. Tong Y, Mentlein R, Buhl R, et al: Overexpression of midkine contributes to anti-apoptotic effects in human meningiomas. J Neurochem 100: 1097-1107, 2007.

46. Friedrich C, Holtkamp N, Cinatl J Jr, et al: Overexpression of Midkine in malignant peripheral nerve sheath tumor cells inhibits apoptosis and increases angiogenic potency. Int J Oncol 27: 1433-1440, 2005

47. Mashour GA, Ratner N, Khan GA, Wang HL, Martuza RL and Kurtz A: The angiogenic factor midkine is aberrantly expressed in NF1-deficient Schwann cells and is a mitogen for neurofibroma-derived cells. Oncogene 20: 97-105, 2001.

48. Choudhuri R, Zhang HT, Donnini S, Ziche M and Bicknell R: An angiogenic role for the neurokines midkine and pleiotrophin in tumorigenesis. Cancer Res 57: 1814-1819, 1997.

49. Kadomatsu K, Hagihara M, Akhter S, Fan QW, Muramatsu H and Muramatsu T: Midkine induces the transformation of NIH3T3 cells. Br J Cancer 75: 354-359, 1997.

50. Tsutsui J, Kadomatsu K, Matsubara S, et al: A new family of heparin-binding growth/differentiation factors: increased midkine expression in Wilms' tumor and other human carcinomas. Cancer Res 53: 1281-1285, 1993.

51. Adachi Y, Reynolds PN, Yamamoto M, et al: Midkine promoter-based adenoviral vector gene delivery for pediatric solid tumors. Cancer Res 60: 4305-4310, 2000.

52. Adachi Y, Reynolds PN, Yamamoto M, et al: A midkine promoter-based conditionally replicative adenovirus for treatment of pediatric solid tumors and bone marrow tumor purging Cancer Res 61: 7882-7888, 2001

53. Ambrosini G, Adida C and Altieri DC: A novel anti-apoptosis gene, survivin, expressed in cancer and lymphoma. Nat Med 3: 917-921, 1997

54. Yoshida H, Ishiko O, Sumi T, Matsumoto Y and Ogita S: Survivin, bcl-2 and matrix metalloproteinase-2 enhance progression of clear cell- and serous-type ovarian carcinomas. Int J Oncol 19: 537-542, 2001.

55. Ikeguchi M, Ueta T, Yamane Y, Hirooka Y and Kaibara N: Inducible nitric oxide synthase and survivin messenger RNA expression in hepatocellular carcinoma. Clin Cancer Res 8 3131-3136, 2002.

56. Ikeguchi $M$ and Kaibara N: Survivin messenger RNA expression is a good prognostic biomarker for oesophageal carcinoma. Br J Cancer 87: 883-887, 2002.

57. Kim PJ, Plescia J, Clevers H, Fearon ER and Altieri DC: Survivin and molecular pathogenesis of colorectal cancer. Lancet 362: 205-209, 2003.
58. Falleni M, Pellegrini C, Marchetti A, et al: Survivin gene expression in early-stage non-small cell lung cancer. J Pathol 200: 620-626, 2003.

59. Kennedy SM, O'Driscoll L, Purcell R, et al: Prognostic importance of survivin in breast cancer. Br J Cancer 88: 1077-1083, 2003

60. Zhu ZB, Makhija SK, Lu B, et al: Targeting mesothelioma using an infectivity enhanced survivin-conditionally replicative adenoviruses. J Thorac Oncol 1: 701-711, 2006.

61. Zhu ZB, Makhija SK, Lu B, et al: Transcriptional targeting of tumors with a novel tumor-specific survivin promoter. Cancer Gene Ther 11: 256-262, 2004

62. Li F and Altieri DC: Transcriptional analysis of human survivin gene expression. Biochem J 344: 305-311, 1999.

63. Li F and Altieri DC: The cancer antiapoptosis mouse survivin gene: characterization of locus and transcriptional requirements of basal and cell cycle-dependent expression. Cancer Res 59: 3143-3151, 1999

64. Bao R, Connolly DC, Murphy M, et al: Activation of cancerspecific gene expression by the survivin promoter. J Natl Cancer Inst 94: 522-528, 2002

65. Lu B, Makhija SK, Nettelbeck DM, et al: Evaluation of tumorspecific promoter activities in melanoma. Gene Ther 12: 330-338, 2005.

66. Nugent CI and Lundblad V: The telomerase reverse transcriptase: components and regulation. Genes Dev 12: 1073-1085, 1998.

67. Nakamura TM, Morin GB, Chapman KB, et al: Telomerase catalytic subunit homologs from fission yeast and human. Science 277: 955-959, 1997.

68. Kyo S, Kanaya T, Takakura M, et al: Expression of human telomerase subunits in ovarian malignant, borderline and benign tumors. Int J Cancer 80: 804-809, 1999

69. Takakura M, Kyo S, Kanaya T, et al: Cloning of human telomerase catalytic subunit (hTERT) gene promoter and identification of proximal core promoter sequences essential for transcriptional activation in immortalized and cancer cells Cancer Res 59: 551-557, 1999.

70. Majumdar AS, Hughes DE, Lichtsteiner SP, Wang Z, Lebkowski JS and Vasserot AP: The telomerase reverse transcriptase promoter drives efficacious tumor suicide gene therapy while preventing hepatotoxicity encountered with constitutive promoters. Gene Ther 8: 568-578, 2001

71. Bilsland AE, Anderson CJ, Fletcher-Monaghan AJ, et al: Selective ablation of human cancer cells by telomerase-specific adenoviral suicide gene therapy vectors expressing bacterial nitroreductase. Oncogene 22: 370-380, 2003

72. Forsyth NR, Wright WE and Shay JW: Telomerase and differentiation in multicellular organisms: turn it off, turn it on, and turn it off again. Differentiation 69: 188-197, 2002.

73. Kotoula V, Hytiroglou P, Pyrpasopoulou A, Saxena R, Thung SN and Papadimitriou CS: Expression of human telomerase reverse transcriptase in regenerative and precancerous lesions of cirrhotic livers. Liver 22: 57-69, 2002.

74. Lee HW, Blasco MA, Gottlieb GJ, Horner JW II, Greider CW and DePinho RA: Essential role of mouse telomerase in highly proliferative organs. Nature 392: 569-574, 1998.

75. Fukazawa T, Maeda Y, Sladek FM and Owen-Schaub LB Development of a cancer-targeted tissue-specific promoter system. Cancer Res 64: 363-369, 2004.

76. Sterman DH, Recio A, Vachani A, et al: Long-term follow-up of patients with malignant pleural mesothelioma receiving highdose adenovirus herpes simplex thymidine kinase/ganciclovir suicide gene therapy. Clin Cancer Res 11: 7444-7453, 2005.

77. Varghese S and Rabkin SD: Oncolytic herpes simplex virus vectors for cancer virotherapy. Cancer Gene Ther 9: 967-978, 2002.

78. Power AT and Bell JC: Taming the Trojan horse: optimizing dynamic carrier cell/oncolytic virus systems for cancer biotherapy. Gene Ther 15: 772-779, 2008.

79. Endter C and Dobner T: Cell transformation by human adenoviruses. Curr Top Microbiol Immunol 273: 163-214, 2004.

80. Vollmer CM, Ribas A, Butterfield LH, et al: p53 selective and nonselective replication of an E1B-deleted adenovirus in hepatocellular carcinoma. Cancer Res 59: 4369-4374, 1999.

81. Heise C, Ganly I, Kim YT, Sampson-Johannes A, Brown R and Kirn D: Efficacy of a replication-selective adenovirus against ovarian carcinomatosis is dependent on tumor burden, viral replication and p53 status. Gene Ther 7: 1925-1929, 2000. 
82. Khuri FR, Nemunaitis J, Ganly I, et al: A controlled trial of intratumoral ONYX-015, a selectively-replicating adenovirus, in combination with cisplatin and 5-fluorouracil in patients with recurrent head and neck cancer. Nat Med 6: 879-885, 2000.

83. Rodriguez R, Schuur ER, Lim HY, Henderson GA, Simons JW and Henderson DR: Prostate attenuated replication competent adenovirus (ARCA) CN706: a selective cytotoxic for prostatespecific antigen-positive prostate cancer cells. Cancer Res 57: 2559-2563, 1997.

84. DeWeese TL, van der Poel H, Li S, et al: A phase I trial of CV706, a replication-competent, PSA selective oncolytic adenovirus, for the treatment of locally recurrent prostate cancer following radiation therapy. Cancer Res 61: 7464-7472, 2001.

85. Van Houdt WJ, Haviv YS, Lu B, et al: The human survivin promoter: a novel transcriptional targeting strategy for treatment of glioma. J Neurosurg 104: 583-592, 2006.

86. Toyoda E, Doi R, Kami K, et al: Midkine promoter-based conditionally replicative adenovirus therapy for midkineexpressing human pancreatic cancer. J Exp Clin Cancer Res 27: 30, 2008.

87. Fukazawa T, Matsuoka J, Naomoto Y, Maeda Y, Durbin ML and Tanaka N: Malignant pleural mesothelioma-targeted CREBBP/EP300 inhibitory protein 1 promoter system for gene therapy and virotherapy. Cancer Res 68: 7120-7129, 2008.

88. Gordon GJ, Rockwell GN, Jensen RV, et al: Identification of novel candidate oncogenes and tumor suppressors in malignant pleural mesothelioma using large-scale transcriptional profiling Am J Pathol 166: 1827-1840, 2005.

89. Wickham TJ, Tzeng E, Shears LL II, et al: Increased in vitro and in vivo gene transfer by adenovirus vectors containing chimeric fiber proteins. J Virol 71: 8221-8229, 1997.

90. Stevenson SC, Rollence M, White B, Weaver L and McClelland A: Human adenovirus serotypes 3 and 5 bind to two different cellular receptors via the fiber head domain. J Virol 69: 2850-2857, 1995.

91. Durmort C, Stehlin C, Schoehn G, et al: Structure of the fiber head of Ad3, a non-CAR-binding serotype of adenovirus. Virology 285: 302-312, 2001

92. Ulasov IV, Tyler MA, Zheng S, Han Y and Lesniak MS: CD46 represents a target for adenoviral gene therapy of malignant glioma. Hum Gene Ther 17: 556-564, 2006.

93. Kanerva A, Mikheeva GV, Krasnykh V, et al: Targeting adenovirus to the serotype 3 receptor increases gene transfer efficiency to ovarian cancer cells. Clin Cancer Res 8: 275-280, 2002

94. Zabner J, Chillon M, Grunst T, et al: A chimeric type 2 adenovirus vector with a type 17 fiber enhances gene transfer to human airway epithelia. J Virol 73: 8689-8695, 1999.

95. Sakurai F, Mizuguchi $H$ and Hayakawa T: Efficient gene transfer into human $\mathrm{CD} 34^{+}$cells by an adenovirus type 35 vector. Gene Ther 10: 1041-1048, 2003.
96. Krasnykh V, Belousova N, Korokhov N, Mikheeva G and Curiel DT: Genetic targeting of an adenovirus vector via replacement of the fiber protein with the phage T4 fibritin. J Virol 75: 4176-4183, 2001.

97. Smith TA, White BD, Gardner JM, Kaleko M and McClelland A: Transient immunosuppression permits successful repetitive intravenous administration of an adenovirus vector. Gene Ther 3: 496-502, 1996.

98.Ilan Y, Jona VK, Sengupta K, et al: Transient immunosuppression with FK506 permits long-term expression of therapeutic genes introduced into the liver using recombinant adenoviruses in the rat. Hepatology 26: 949-956, 1997.

99. Ye X, Robinson MB, Pabin C, Batshaw ML and Wilson JM: Transient depletion of CD4 lymphocyte improves efficacy of repeated administration of recombinant adenovirus in the ornithine transcarbamylase deficient sparse fur mouse. Gene Ther 7: 1761-1767, 2000 .

100. Chirmule N, Raper SE, Burkly L, et al: Readministration of adenovirus vector in nonhuman primate lungs by blockade of CD40-CD40 ligand interactions. J Virol 74: 3345-3352, 2000.

101. O'Riordan CR, Lachapelle A, Delgado C, et al: PEGylation of adenovirus with retention of infectivity and protection from neutralizing antibody in vitro and in vivo. Hum Gene Ther 10: 1349-1358, 1999.

102. Fisher KD, Stallwood Y, Green NK, Ulbrich K, Mautner V and Seymour LW: Polymer-coated adenovirus permits efficient retargeting and evades neutralising antibodies. Gene Ther 8: 341-348, 2001

103. Fisher KD, Ulbrich K, Subr V, et al: A versatile system for receptor-mediated gene delivery permits increased entry of DNA into target cells, enhanced delivery to the nucleus and elevated rates of transgene expression. Gene Ther 7: 1337-1343, 2000.

104. Vogels R, Zuijdgeest D, van Rijnsoever R, et al: Replicationdeficient human adenovirus type 35 vectors for gene transfer and vaccination: efficient human cell infection and bypass of preexisting adenovirus immunity. J Virol 77: 8263-8271, 2003.

105. Holterman L, Vogels R, van der Vlugt R, et al: Novel replication-incompetent vector derived from adenovirus type 11 (Ad11) for vaccination and gene therapy: low seroprevalence and non-cross-reactivity with Ad5. J Virol 78: 13207-13215, 2004.

106.Parks RJ, Chen L, Anton M, Sankar U, Rudnicki MA and Graham FL: A helper-dependent adenovirus vector system: removal of helper virus by Cre-mediated excision of the viral packaging signal. Proc Natl Acad Sci USA 93: 13565-13570, 1996.

107. Roberts DM, Nanda A, Havenga MJ, et al: Hexon-chimaeric adenovirus serotype 5 vectors circumvent pre-existing antivector immunity. Nature 441: 239-243, 2006. 